\title{
Conocimientos y actitudes de los padres acerca del trastorno por déficit de atención e hiperactividad (TDAH) en un colegio de Independencia, Lima, Perú.
}

\author{
Knowledge and attitudes of parents towards attention deficit and hyperactivity disorder (ADHD) in a school \\ of Independencia, Lima, Perú.
}

José López-Rodas', Bruno Alvarado-Broncano², Beltran Huerta-Lovaton².

\begin{abstract}
RESUMEN
Objetivos: Evaluar los conocimientos y actitudes de los padres acerca del trastorno por déficit de atención e hiperactividad (TDAH), en un colegio del distrito de Independencia. Material y métodos: Se realizó un estudio de tipo descriptivo, con una muestra representativa de 255 padres de familia de una población de 1200, de un colegio del distrito de Independencia de la ciudad de Lima. Se aplicó un cuestionario estructurado basado en experiencias anteriores previa validación del mismo. Resultados: El 76,4\% de los padres escucharon hablar sobre TDAH. El 62,7\% de los padres están de acuerdo con que es un problema importante en el niño, 68,6\% de los padres dijo que las malas prácticas de crianza y maltrato de los padres es la causa del TDAH, 87,5\% de los padres consideran que los niños con este trastorno experimentan más dificultades conductuales y emocionales que sus compañeros de clase, 74,5\% consideró que estos niños requieren tratamiento médico. Conclusiones: Los datos muestran diferencias en los conocimientos y actitudes de los padres respecto al TDAH, lo cual es importante tener en cuenta al momento de cualquier intervención de promoción de la salud respecto a este problema. Algunas de las diferencias parecen ser semejantes a las encontradas en estudios similares. (Rev Neuropsiquiatr 2010;73:45-51).
\end{abstract}

PALABRAS CLAVE: TDAH, conocimiento, atención, actitud.

\section{SUMMARY}

Objectives: To evaluate the knowledge and attitudes of parents towards attention deficit and hyperactivity disorder (ADHD) in a school of Independencia, Lima. Material and methods: We conducted a descriptive study with a representative sample of 255 parents from a total population of 1200. A structured questionnaire based on previous experiences prior validation was applied. Results: $76.4 \%$ of parents had heard about ADHD, $62.7 \%$ agree that is a major problem in children, $68.6 \%$ said that poor parenting practices and abuse by parents is the cause of ADHD, and

1 Médico - Psiquiatra. Universidad Peruana Cayetano Heredia. Instituto Nacional de Salud Mental Honorio Delgado - Hideyo Noguchi. Lima, Perú.

2 Bachiller en Medicina. Universidad Peruana Cayetano Heredia. Lima, Perú. 
87.5\% believe that children with this disorder experience more behavioral and emotional difficulties in relation to their classmates. Seventy four percent think that these children require medical treatment. Conclusions: The data show differences in the considerations of parents about ADHD, which is important to keep in mind when promoting any action on this health problem. Some of the differences appear to be similar to those found in similar studies. (Rev Neuropsiquiatr 2010;73:45-51).

KEY WORDS: ADHD, knowledge, attention, attitude.

\section{INTRODUCCIÓN}

El trastorno por déficit de atención e hiperactividad (TDAH) se caracteriza porque el paciente para la etapa del desarrollo tiene tres síntomas básicos: deficiencia en la atención, impulsividad e hiperactividad inusual (1-5). El TDAH es un trastorno muy frecuente, que ocurre en el 5 a $10 \%$ de los niños de etapa escolar primaria; constituyendo el mayor problema para la escuela de ese nivel de educación.

La percepción pública del TDAH, es abundante en mitos, conceptos e información errónea sobre sus causas, significado, diagnóstico y tratamiento del trastorno. Algunos estudios realizados, muestran que el TDAH para diversas poblaciones no es considerado un trastorno, más bien es visto como un problema benigno, y su presentación hasta es considerada en exceso $(6,7)$. Otros estudios en los que se evalúan los conocimientos y actitudes sobre el TDAH por profesores, padres y niños, hacen notar que los primeros tienden a magnificar el problema (8).

Los diversos estudios sobre este trastorno, indican claramente que el TDAH tiende a darse en familias, y que los patrones de transmisión son en gran parte genéticos $(9,10)$. Más de 20 estudios genéticos han mostrado evidencia de que el TDAH tiene un fuerte componente hereditario. Sin embargo, se está más de acuerdo en que el TDAH es un trastorno complejo que aparentemente resulta de múltiples factores en los que se sospecha la interacción de múltiples genes, un bajo peso al nacer, tabaquismo materno prenatal y otros problemas prenatales adicionales que podrían contribuir a otros casos de TDAH $(11,12)$.

Es importante destacar algunas de las entidades clínicas que se asocian con gran frecuencia al TDAH: los trastornos de ansiedad. Alrededor de un 25\% de estos niños son más susceptibles de padecer problemas de ansiedad al resultarles difícil responder de forma adecuada a las exigencias de su entorno. Se señala que los trastornos del estado de ánimo se dan en el 30\%.
Estos tipos de asociación empeoran el pronóstico a mediano y largo plazo, y complican la respuesta terapéutica (8).

Los problemas principales que conlleva un TDAH no tratado adecuadamente son: la pérdida de interés en los estudios y fracasos académicos, el riesgo de drogadicción y delincuencia, las frustraciones laborales, las dificultades sociales y matrimoniales; constituyendo un problema de salud pública tal como ha sido declarado así en los Estados Unidos de Norteamérica (13). Aparte de lo señalado, se requiere sensibilizar a la población a fin de que el TDAH sea atendido lo más tempranamente posible. Ello justifica el presente estudio en razón que al contar con las nociones o ideas que pueden tener los padres de familia respecto a este problema se facilitarían las intervenciones promocionales y preventivas acerca del TDAH. Consideramos que la educación de los padres es un punto de partida para un mejor diagnostico, manejo y tratamiento de estos niños.

El objetivo del presente estudio fue evaluar los conocimientos y actitudes de los padres acerca del TDAH en un colegio del distrito de Independencia en Lima.

\section{MATERIAL Y MÉTODOS}

Se realizó un estudio de tipo descriptivo, transversal con una muestra de 255 padres o apoderados de los alumnos del nivel primario inscritos en el año escolar 2010 que representan a una población de 1200 padres de un colegio público en el distrito de IndependenciaLima. Para la muestra se tomó en cuenta el estudio "Educación y consejería a los padres de los niños con déficit de atención e hiperactividad”, del cual se obtuvo como dato referencial que el $70 \%$ de los padres cuentan con escasa información o desconocen sobre el tema (14). A ellos les fue aplicado un cuestionario estructurado (Anexo 1) en compañía de uno de los autores en los ambientes del colegio al momento que ingresaban o recogían a su hijo del centro educativo, no siendo este proceso de muestreo de forma aleatoria, 
realizándose en un periodo de 2 semanas. Este cuestionario evalúo conocimientos y actitudes en relación al trastorno por déficit de atención e hiperactividad, así como algunos datos demográficos; previo consentimiento informado de su participación por escrito. Los criterios de inclusión fueron los padres o apoderados de niños matriculados en el nivel primario del año escolar 2010, siendo los de exclusión aquellos padres o apoderados cuyos hijos presenten diagnóstico previo de déficit de atención e hiperactividad.

El instrumento aplicado fue diseñado a partir de un cuestionario de un estudio previo realizado en Irán sobre TDAH (15), desarrollado originalmente en inglés. Nuestro estudio, incluyó la validación del instrumento por dos expertos en el conocimiento integral del idioma

\section{Anexo 1. Cuestionario sobre conocimiento y actitud de los padres acerca del trastorno por déficit de atención e hiperactividad (TDAH).}

1. El Trastorno por déficit de atención e hiperactividad se debe a una causa hereditaria

Verdadero Falso

2. El Trastorno por déficit de atención e hiperactividad en un niño tiene como una de sus características ser independiente $\quad$ Verdadero Falso

3. El Trastorno por déficit de atención e hiperactividad en un niño tiene como una de sus características ser curioso $\quad$ Verdadero Falso 4. El Trastorno por déficit de atención e hiperactividad en un niño tiene como una de sus características ser más inteligente

Verdadero Falso

5. El Trastorno por déficit de atención e hiperactividad es un problema importante para el niño $\quad$ Verdadero Falso

6. El Trastorno por déficit de atención e hiperactividad mejorará espontáneamente sólo en $\begin{array}{ll}\text { algunos casos } & \text { Verdadero Falso }\end{array}$ 7. El Trastorno por déficit de atención e hiperactividad puede ser causado por la mala práctica de crianza y maltrato de los padres $\quad$ Verdadero Falso 8. Los niños con trastorno por déficit de atención e hiperactividad están en un riesgo más alto de ausentismo escolar y escaparse de clase $\quad$ Verdadero Falso 9. Las dificultades relacionadas con el trastorno por déficit de atención e hiperactividad sin tratamiento persisten para toda la vida en la mayoría de los casos $\quad$ Verdadero Falso 10. Los niños con Trastorno por déficit de atención e hiperactividad deben recibir educación especial, lo cual sería más favorable Verdadero Falso 11. Los niños con Trastorno por déficit de atención e hiperactividad tienen un alto riesgo de convertirse en delincuentes al ser adolescentes Verdadero Falso 12. Los niños con Trastorno por déficit de atención e hiperactividad tienen inteligencia más alta que los niños sin TDAH Verdadero Falso 13. Las mismas reglas de conducta deben aplicarse por igual a los niños con trastorno por déficit de atención e hiperactividad como con los niños que no lo tienen Verdadero Falso 14. Los niños con trastorno por déficit de atención e hiperactividad experimentan más dificultades conductuales y emocionales en relación a sus compañeros de clase

Verdadero Falso 15. El rendimiento escolar de los niños con Trastorno por déficit de atención e hiperactividad es_menor que el de sus compañeros sin TDAH Verdadero Falso 16. Los niños con Trastorno por déficit de atención e hiperactividad experimentan más dificultades en sus relaciones con los miembros de su familia $\quad$ Verdadero Falso 17. Los estudiantes con Trastorno por déficit de atención e hiperactividad deben recibir menos tareas que los niños sin ese problema Verdadero Falso 18. Los estudiantes con Trastorno por déficit de atención e hiperactividad deben ser evaluados de forma oral Verdadero Falso 19. El Trastorno por déficit de atención e hiperactividad puede ser tratado con medicación adecuada Verdadero Falso 20. El Trastorno por déficit de atención e hiperactividad a menudo puede ser causado por el azúcar u otros aditivos alimentarios Verdadero Falso 
inglés, a través de la traducción y revisión del mismo; la versión en castellano fue revisada y traducida nuevamente al inglés por otro traductor, con la finalidad de asegurar que reflejara realmente el instrumento inicial, haciendo luego la comparación de ambas versiones, y realizando las modificaciones necesarias para su plena comprensión.

Posteriormente se conformó un grupo focal constituido por 6 padres de familia, escogidos al azar y al que se les aplicó el instrumento con el fin de evaluar la claridad y el contenido de las preguntas, realizándose las correcciones que surgieron de dicho procedimiento. Una vez realizado esto, se procedió a la realización de una prueba piloto a 40 padres pertenecientes al colegio para la validación interna a través del método de coeficiente biserial-puntual. Se desestimaron 3 ítems de la encuesta original al no obtener el puntaje necesario de aceptación del área de conocimientos y actitudes, el resto cumplió con un puntaje adecuado para este fin, por consiguiente en este caso el instrumento tiene validez interna. La confiabilidad se llevó a cabo mediante la fórmula 20 de Kuder - Richardson siendo este mayor de a 0,60 , el cual se considera aceptable; por consiguiente, el instrumento es confiable. El cuestionario final constó de 25 preguntas divididas en 2 secciones; la primera abarca los datos generales del encuestado, la segunda evalúa los conocimientos y actitudes a tomar por los padres, sobre el trastorno por déficit de atención e hiperactividad, siendo las respuestas de carácter dicotómico (Verdadero/Falso).

Los datos fueron revisados e ingresados a la base de datos en el programa SPSS versión 13.0, luego se realizaron los respectivos análisis, dependiendo de las variables establecidas y los propósitos del estudio. Una vez establecidos los resultados de acuerdo a su distribución porcentual y de frecuencias fueron agrupados en tablas.

\section{RESULTADOS}

En un periodo de 2 meses, fueron entregados 255 cuestionarios a igual número de padres de familia de un centro educativo primario del colegio público del distrito de Independencia-Lima, quienes habían aceptado participar de manera voluntaria. Las edades de los padres de familia oscilaron entre 18 y 58 años, con una media de $34,12 \pm 2,21$ con predomino el sexo femenino (98,8\%). El grado de instrucción de estos padres tuvo predominio del nivel secundario en $72,5 \%$, seguido del grado superior con 19,2\% y de nivel primario con 8,2\%. El 79,6\%, refirió ser ama de casa,
$18,8 \%$ empleados y 1,6\% estudiantes. Del total considerado, 12,5\% de los encuestados, señaló haber obtenido información sobre el TDAH a través de personal de salud, 23,5\% negó conocer acerca de este trastorno y 63,9\% dijo haber obtenido información por otros medios.

Un 47,5\% de los padres señaló que el TDAH tenía una causa hereditaria. El 47,8\% respondieron que los niños con TDAH denotan independencia. El 73,7\%, respondieron que tener TDAH es signo de curiosidad y 44,7\% de ellos contestaron que los síntomas del TDAH son signos de una inteligencia superior. El 62,7\% de los padres están de acuerdo en que es un problema importante en el niño y $42 \%$ respondieron que mejora espontáneamente sólo en algunos casos. El 68,6\% de los padres dijo que las malas prácticas de crianza y maltrato de los padres es la causa del TDAH. El 74,5\% cree que el TDAH aumenta el riesgo de ausentismo escolar y el $48,2 \%$ de ellos cree que las dificultades relacionadas con este trastorno sin tratamiento persisten para toda la vida.

Un 75,3\% respondieron que estos niños deberían recibir educación especial. El 73,3\% de los encuestados contestaron que los niños tienen un alto riesgo de convertirse en delincuentes al ser adolescentes. Un $39,6 \%$ de ellos respondieron que los niños con este trastorno tienen una inteligencia superior a los niños que no lo padecen. El 36,7\% respondieron que la misma disciplina y normas utilizadas para todos los niños deberían aplicarse a los niños con TDAH. Un 87,5\% de los padres respondieron que los niños con este trastorno experimentan más dificultades conductuales y emocionales en relación a sus compañeros de clase.

El $70,2 \%$ de los padres, respondieron que el rendimiento escolar es bajo en estos niños. El 82,7\% de los padres de familia respondieron que estos niños experimentan más dificultades en la relación con su familia. Un $22 \%$ respondieron afirmativamente que estos niños deben recibir menos tareas; 49,4\% respondieron que los niños que padecen este problema deberían ser evaluados de forma oral. Con respecto al manejo, un $74,5 \%$ consideró que estos niños requieren tratamiento médico. Un 16,1\% opinó que este trastorno se podía deber al consumo de azúcar u otros alimentos.

\section{DISCUSIÓN}

El TDAH es una patología de alta prevalencia en la niñez. En los países donde se ha generado un sistema de promoción de salud importante para ofrecer una 
Conocimientos y actitudes de los padres acerca del trastorno por déficit de atención e hiperactividad.

atención precoz se ha observado la generación de incremento de la atención de los niños con este tipo de características, lo que permite notar la gran ansiedad familiar y preocupación escolar que este trastorno provoca.

El conocer las creencias que tiene la población sobre el TDAH, permite mejorar la información a ofrecer durante la relación médico paciente en los programas de atención primaria. Es importante mencionar primero que el instrumento utilizado no pretende ser un instrumento diagnóstico, sino mas bien, una referencia de lo que la gente cree o la actitud que tienen respecto al TDAH. Queremos enfatizar que el conocimiento de los padres sobre TDAH juega un rol importante como se ha observado en otros estudios, teniendo un efecto positivo sobre la salud mental de estos al poder tomar decisiones sobre sus hijos que saben que podrán beneficiarlos $(16,17)$. Estudios acerca de los conocimientos y actitudes de los padres son extremadamente limitados $(18,19)$. Insistimos en que los datos obtenidos en la presente investigación deben ser usados en la atención primaria.

Que el 76,4\% de la población encuestada haya escuchado hablar de TDAH a través de medios de comunicación, señalando mayoritariamente a la televisión y la radio, podría hacernos suponer que no se requiere de mucha exigencia para realizar programas de promoción de la salud acerca del TDAH, al considerar que la población se encuentra de alguna forma informada acerca de este trastorno o a lo que la CIE 10 o en el DSM IV describen como TDAH; pero ello es una ligereza puesto que en los últimos años se ha originado gran controversia sobre el tema entre connotados profesionales, que se ha desplazado a la opinión pública en general. Un ejemplo de esto, es el estudio "Educación y consejería de los padres de los niños con trastorno por déficit de atención e hiperactividad”, donde encuentran que la mayoría de los padres señala no tener suficiente información, y aunque la mayoría de padres también ha oído hablar de TDAH parece que no son conscientes totalmente de ello (14); esto hace pensar que si bien en esta población que estudiamos, la mayor parte ha oído sobre este tema, la calidad de su información no necesariamente puede ser buena, eso dependerá muchas veces de la cantidad y calidad de información a la que sean expuestos los padres y al grado de comprensión que estos tengan. Algo que llama la atención es que sólo 12\% refirió haber recibido información por personal de salud, lo que puede reflejar que las campañas de promoción de la salud con respecto al TDAH en colegios son insuficientes o casi nulas.

Con respecto a las posibles causas desencadenantes del TDAH, el 47\% de la población encuestada consideró que existe una predisposición hereditaria en la presentación del TDAH, y una cantidad mayor (69\%) consideró que era provocado por malas prácticas de crianza y maltrato de los padres. Cabe resaltar que al igual que en nuestra población en estudios previos la mayoría de padres son reacios a aceptar las dificultades de su hijo como un trastorno (20) y más bien se atribuya a un problema en la formación educativa del niño. Esto es importante ya que puede ser una de las causas por las cuales los padres no acuden a los centros de salud en busca de ayuda y retrasan el diagnóstico que puede llevar al desarrollo de graves consecuencias posteriores en el menor, ya que al ignorar el origen del problema pueden contribuir a empeorar más los síntomas de los niños que lo padecen. Sumado a esto puede haber un sentimiento de insatisfacción o culpa de los padres por el comportamiento de sus hijos, que puede desaparecer cuando aprenden sobre el TDAH (21). Esto nos hace pensar que en la atención primaria se debe considerar el reforzar y hacer llegar de forma hábil la información sobre el TDAH, sin descuidar los aspectos psicológicos relacionados a este.

En relación a las preguntas asociadas a signos o características del TDAH, un 74\% de los padres consideran que el ser curioso es un signo de TDAH, sumado a un $48 \%$ y un $45 \%$ que consideran que la independencia y el ser más inteligente respectivamente, también lo son. Esto nos hace notar que para un gran número de padres el concepto de TDAH no es muy claro, lo cual dificultaría un manejo adecuado de los niños que lo padezcan. Como se ha descrito en trabajos realizados, los primeros signos del TDAH no suelen reconocerse hasta los primeros años de colegio y el diagnóstico y tratamiento correctos suelen demorar de uno a varios años $(6,8)$. Por lo cual, informar adecuadamente a los padres debería ser una meta por parte de las entidades educativas como de las instituciones de salud.

Alrededor de las dos terceras partes de los encuestados consideran el TDAH como un problema importante en el niño, esto refleja la preocupación por parte de los padres de que el TDAH es un problema que afecta la formación de sus hijos, sin embargo hay muchos padres que aún ignoran las consecuencias o implicancias de esta patología en el niño; esto es 
importante destacar ya que este grupo de padres pueden desestimar las consecuencias del TDAH a posteriori lo cual se puede traducir en años de incapacidad para un menor al no tener un diagnóstico y no tomar tratamiento. Ya que las creencias y actitudes de los padres se relacionan directamente con la decisión de buscar algún servicio de salud mental para los niños con TDAH (21), y que el nivel aceptable de actividad para los niños es fijado por expectativas culturales (22), lo que puede afectar la forma de cómo evaluar y reportar el comportamiento de sus niños (23); es de importancia promover la implementación de acciones conjuntas entre los servicios de salud y los colegios, a fin de poder lograr una atención primaria que permita facilitar la mejor interacción entre personal de salud, profesores y padres en la solución de este problema.

Algo importante a considerar es el hecho de que al parecer la mayor parte de los padres asocian el TDAH con incapacidad mental o un problema de desinterés por los estudios, esto se ve reflejado en que la mayor parte de los padres muestra afinidad por optar por alguna actitud que promueva menores exigencias así como estimulación de la persona en el caso de incapacidad o una actitud más estricta por parte de los maestros como mayores exigencias académicas en el caso de problema de desinterés por los estudios. Esto es importante ya que si bien la mayor parte de los padres ha escuchado sobre TDAH al parecer no distinguen que se trate de un problema de discapacidad que por supuesto requiere medicación y una etapa de nivelación académica para poder superar estas deficiencias, lo que hace notar la desinformación o la información errónea que también se da en estos aspectos en los que no sólo se ven implicados los padres sino también los maestros que acceden a estas peticiones estando desinformados sobre el tema. Por lo que teniendo en cuenta la ley de inclusión del alumno discapacitado del sector educación en nuestro país, se podría facilitar las acciones para que los profesores reciban una mayor formación en el manejo del niño con este problema.

Con respecto al manejo, $74 \%$ de los encuestados consideran que el TDAH puede ser tratado con la medicación adecuada, esto es muy positivo ya que la mayor parte asume que se trata de una enfermedad y requiere tratamiento, sin embargo aún hay una parte que al parecer no entiende esto, que podría ser una consecuencia de lo que se ha visto en otros estudios, en que los profesores o psicólogos escolares atribuyen las dificultades a problemas familiares, emocionales, poca motivación, etc.; y cuando el diagnóstico finalmente se efectúa, no es raro que se opongan al tratamiento medicamentoso, diciendo que no es tan severo el problema como para justificarlo, o que la medicación es peligrosa (6). Así cabe resaltar también que un $42 \%$ asume que el TDAH se puede resolver espontáneamente sólo en algunos casos, lo cual coincide con los conocimientos actuales de que un $25 \%$ de estos pacientes mejoran espontáneamente, sin embargo esto no debe impedir el reforzar el conocimiento y comprensión sobre el TDAH en los padres, profesores y personal médico a través de los centros educativos y puestos de salud con el fin de mantener y mejorar el manejo de estos niños.

Dentro de las limitaciones del presente estudio, tenemos que no se encuestó a la pareja de padres, sino sólo al que se presentó en el colegio, que fue predominantemente del sexo femenino. Esto podría ser tomado en cuenta en futuros trabajos de este tipo para poder evaluar si la pareja de padres comparte las mismas opiniones o conceptos y si esto produce algún efecto en la toma de decisiones.

Así también pudo ser positivo conocer si el colegio cuenta con personal de salud que promueva la enseñanza a través de charlas.

Otro punto que podría haberse tomado en consideración hubiera sido el estrato socio-económico, ya que como mencionamos aparentemente los medios de comunicación como la televisión o radio son los que más difunden el conocimiento a los padres.

A pesar de que muchos padres han oído hablar del TDAH y manejan en promedio un conocimiento bueno en este estudio, aún existe una necesidad de mejorar la comprensión y el entendimiento de los padres sobre el TDAH (concepción del problema, su causa, su efecto en el comportamiento y la educación y su impacto social) a través de programas de promoción de la salud a nivel de los centros educativos. Es importante valorar también que el instrumento validado en este estudio podrá ser empleado como una herramienta de asistencia promocional y ser aplicado en diversos grupos poblacionales con el fin de conocer de cierta forma las limitaciones de las poblaciones en sus conocimientos sobre el TDAH, a fin de poder capacitarlas adecuadamente lo cual será un punto de partida para un mejor diagnóstico, manejo y tratamiento de estos niños como antes lo hemos señalado. 
Conocimientos y actitudes de los padres acerca del trastorno por déficit de atención e hiperactividad.

\section{Correspondencia:}

Bruno Alvarado Broncano.

Las Amatistas 424-C, Balconcillo.

Lima 13, Peru.

Correo electrónico: alvacanobruno@hotmail.com

\section{REFERENCIAS BIBLIOGRÁFICAS}

1. American Psychiatric Association. Diagnostic and statistical manual of mental disorders: DSM-IV. 4th ed., text revision. Washington D.C.: American Psychiatric Association; 2000.

2. Barbaresi W, Katusic S, Colligan R, Pankratz S, Weaever A, Weber K, et al. How common is AttentionDeficit/Hyperactivity Disorder? Arch Pediatr Adolesc Med 2002; 156(3):217-224.

3. Leibson C, Katusic S, Barbaresi W, Ransom J, O'Brien P. Use and costs of Medical Care for children and adolescents with and without Attention-Deficit/ Hyperactivity Disorder. JAMA 2001; 285(1): 60-66.

4. Surgeon General of the United States. Mental Health: A report of the surgeon general. Rockville, MD: U.S. Department of Health and Human Services; 1999.

5. American Academy of Pediatrics. Clinical practice guidelines: Diagnosis and evaluation of the child with attention-deficit/hyperactivity disorder. Pediatrics 2000; 105: 1158-1170.

6. Filomeno A. El niño con déficit de atención o hiperactividad: cómo pasar del fracaso al éxito. 2da edición. Lima: Universidad Peruana Cayetano Heredia; 2009.

7. Teeter P. Myths and misconceptions about ADHD: Science over Cynicis. Attention! 2003: 27 - 29.

8. Castro J. Psiquiatría de niños y adolescentes. Primera edición. Lima: Universidad Peruana Cayetano Heredia; 2009.

9. Tannock R. Attention deficit hyperactivity disorder: Advances in cognitive, neurobiological, and genetic research. J Child Psychol Psychiatry 1998; 39: 65-99.

10. Swanson JM, Castellanos FX. Biological bases of ADHD: Neuroanatomy, genetics, and pathophysiology. In: Jensen PS, Cooper JR (eds). Attention deficit hyperactivity disorder: State of the science, best practice. New Jersey: Civic Resarch Institute; 2002.p.720.

11. Connor DR. Preschool attention deficit hyperactivity disorder: A review of prevalence, diagnosis, neurobiology, and stimulant treatment. J Dev Behav Pediatr 2002; 23 (S1): S1-S9.

12. Wilens TE, Biederman J, Brown S, Tanguay S, Monteaux MC, Blake C, et al. Psychiatric co-morbidity and functioning in clinically referred preschool children and school age youths with AD/HD. J Am Acad Child Adolesc Psychiatrity 2002;4(3): 26-28.

13. Filomeno A. El trastorno por déficit de atención con hiperactividad (TDAH). Gestión Médica. Lima, Peru: 2005, febrero 21-27 Sección Neurología. p. 19.

14. Ghanizadeh A. Educating and counseling of parents of children with attention-deficit hyperactivity disorder. Patient Educ Couns 2007; 68: 23-28.

15. Ghanizadeh A, Bahredar MJ, Moeini SR. Knowledge and attitudes towards attention deficit hyperactivity disorder among elementary school teachers. Patient Educ Couns 2006; 63: 84-8.

16. Ghanizadeh A, Shahrivar FZ. The effect of parent management training on children with attention deficit hyperactivity disorder. J Child Adoles Ment Health 2005;17:31-4.

17. Corkum P, Rimer P, Schachar R. Parental knowledge of attention-deficit hyperactivity disorder and opinions of treatment options: impact on enrollment and adherence to a 12-month treatment trial. Can J Psychiat 1999;44:1043-8.

18. Chavira V, Lopez SR, Blacher J, Shapiro J. Latina mothers' attributions, emotions, and reactions to the problem behaviors of their children with developmental disabilities. J Child Psychol Psyc 2000;41:245-52.

19. Livingston R. Cultural issues in diagnosis and treatment of ADHD. J Am Acad Child Adol Psych 1999;38:1591-4.

20. Wilcox CE, Washburn R, Patel V. Seeking help for attention deficit hyperactivity disorder in developing countries: A study of parental explanatory models in Goa, India. Soc Sci Med 2007; 64(8):1600-10.

21. Gidwani PP, Opitz GM, Perrin JM. Mothers' views on hyperactivity: a cross-cultural perspective. J Dev Behav Pediatr 2006;27:121-6.

22. Perry CE, Hatton D, Kendall J. Latino parents' accounts of attention deficit hyperactivity disorder. J Transcult Nurs 2005;16:312-21.

23. Pachter LM, Dworkin PH. Maternal expectations about normal child development in 4 cultural groups. Arch Pediat Adol Med 1997;151:1144-50. 